\title{
Nurses and COVID-19
}

\author{
Siniša Franjić \\ Independent Researcher, Bosnia \& Herzegovina
}

\begin{abstract}
The virus which causes COVID-19 is most often transmitted through droplets that occur when an infected person coughs, sneezes or exhales. These droplets are too heavy to fly through the air and fall quickly to the floor and other surfaces. A person can get it by touching his eyes, nose or mouth after touching such contaminated surfaces or by inhaling the virus if he is in the immediate nearness of a person who has COVID-19. The aim of this paper is to draw attention to the hard work of nurses who are faced daily with the most severe forms, but also the most severe consequences for the health of patients during the current pandemic crisis. Nurses are making tremendous efforts to help patients recover as soon as possible. Unfortunately, there are a lot of patients whose health is deteriorating due to the pandemic, despite the enormous efforts of medical professionals who are doing their best to help them. Despite the difficult situation, the announcement of the coming vaccines still offers hope.
\end{abstract}

\section{Keywords}

COVID-19, Virus, Infection, Nurses

\section{Introduction}

Coronavirus are unsegmented single-stranded positive-strand RNA viruses [1]. They belong to the order Nidovirales, the family Coronaviridae, and the subfamily Orthocoronavirinae, which is divided into $\alpha, \beta, \gamma$, and $\delta$ genera according to their serotypic and genomic characteristics. Coronaviruses belong to the genus Coronavirus of the family Coronaviridae. It is named after the wreath-shaped protrusions on the envelope of the virus.

Coronaviruses have an envelope encasing the RNA genome, and the virions (the whole viruses) are round or oval, often polymorphic, with a diameter of 50 to $200 \mathrm{~nm}$. The novel coronavirus is 60 to $140 \mathrm{~nm}$ in diameter. The spike protein is located on the surface of the virus and forms a rodlike structure. As one of the main antigenic proteins of the virus, the spike protein is the main structure used for typing. The nucleocapsid protein encapsulates the viral genome and can be used as a diagnostic antigen.

Viruses generally can survive for several hours on smooth surfaces. If the temperature and humidity permit, they can survive for several days. The novel coronavirus is sensitive to ultraviolet rays and heat. Sustained heat at $132.8{ }^{\circ} \mathrm{F}$ for 30 minutes, ether, $75 \%$ alcohol, chlorine containing disinfectants, peracetic acid, chloroform, and other lipid solvents can effectively inactivate the virus. Chlorhexidine (also known as chlorhexidine gluconate) also effectively inactivates the virus.

In the context of viruses, once these chains of DNA or RNA are released into the cell they can behave in a number of ways [2]. They may do nothing at all. They may kill the cell, having forced its machinery to make copies of itself. They may remain in the cell but redirect that same machinery to their own reproductive ends without killing it. In the case of some common viral diseases - chicken pox, measles - the virus may reactivate months or even decades later. Some viruses may so disrupt the reproductive machinery of the cell that they cause cancer. However, some may also become latent and incorporate permanently into the genes of the host. In the case of some viruses this incorporation may be harmless, or even beneficial, and as we have said, it can be passed from mother to offspring. These properties of viruses explain much of their capacity to cause epidemics. Their similarity to us, and the fact that we are partly composed of material indistinguishable from viruses, makes us sitting ducks for viral assault.

The freedom from the need to fuel and monitor their own reproduction allows viruses one much more crucial property. It permits a much greater capacity for mutation. Higher organisms that reproduce for themselves need to ensure faithful copying of their blueprint into the next generation. Defective cells will not function properly, and the animal may die as a consequence. They - we - therefore rely on a 'proof-reading' mechanism. The chances of a mutational error creeping

*Corresponding author: Dr. Siniša Franjić, Independent Researcher, Bosnia \& Herzegovina

Accepted: February 10, 2021

Published online: February 12, 2021

Citation: Franjić S (2021) Nurses and COVID-19. J Nurs Pract 4(1):232-236 
in when your and my cells reproduce is lower than the order of one in a billion. Viruses are far sloppier. So long as they can enter a cell and reproduce they are 'happy'. A single virus may produce many thousands of offspring in a single cell.

\section{Viruses}

Viruses can be divided into two groups in terms of their surface architectures: A) Enveloped viruses that have an envelope derived from the double-layered lipid cell membrane with virus envelope proteins protruding into it and, B) Non-enveloped viruses that do not have a envelope, with only a viral protein core [3]. Infection of virus starts from virus entry into the host cells to deliver its genome for replication and new virus assembly. In enveloped viruses, the virus entry works by virus-cell membrane fusion, which takes place either in the cell-surface membrane (e.g., human immunodeficiency virus, HIV, takes this way) or in the endosome inside the cell after the virus is taken into the cells through endocytosis (e.g., influenza virus goes through this way).

Virus-cell fusion is mediated by protein-protein or protein-ligand (non-protein molecules) interaction in the interface of virus and cell. On the virus surface there exist several surface proteins, named envelope proteins. The numbers of the envelope proteins on the virus surface depend on different kinds of viruses from different virus families. Their functions can be different, but at least one or two, in general, are involved in virus fusion. Those proteins are usually named as fusion proteins - for example, gp160 in HIV or S protein in severe acute respiratory syndrome coronavirus (SARS-CoV). Envelope proteins bind to their cell-surface receptors, either protein (e.g., CD4 as HIV virus receptor) or other molecules (e.g., sialic acid as infl uenza virus receptor). There is a prominent phenomenon, called binding-induced conformational changes, in the envelope protein during the virus fusion process. This is referred to as the envelope protein conformational changes induced by its binding to the cellular receptors. This conformational change is the driving force to initiate the virus-cell fusion. Research on the envelope protein-receptor binding and the induced conformational change in the recent years on different enveloped viruses has led to some important discovery for us to understand the process of virus fusion and entry. This also leads to several concepts to elucidate the molecular mechanism involved and development for the new antiviral drugs, including some polypeptides.

\section{Coronaviruses}

Coronaviruses are a large family of viruses that causes not only the common cold but also more serious respiratory illnesses [4]. They are a large family of viruses that are common in people and many different species of animals, including camels, cattle, cats, and bats. Rarely, animal coronaviruses can infect people and then spread between people such as with MERSCOV, SARS-COV, and now with this new virus (named SARS-COV-2). The novel coronavirus (2019-nCOV) was identified as the source of the illnesses on January 7 , with the infection traced to Wuhan seafood market that also sold live animals. The outbreak was first observed on December 31, 2019. The virus has been named "SARS-COV-2" and the disease it causes has been named "coronavirus disease 2019" (abbreviated "COVID-19"). The SARS-CoV-2 virus is a betacoronavirus, like MERS-CoV and SARS-CoV. All three of these viruses have their origins in bats.

2019-nCOV emerged in a seafood and poultry market in the Chinese city of Wuhan in 2019. Cases have been detected in most countries worldwide, and on March 11, 2020, the World Health Organization characterized the outbreak as a pandemic. Human-to-human transmission occurs through close contact. Most people get infected with human strains of coronaviruses at some point in their lives. Human coronaviruses can cause other illnesses, such as pneumonia or bronchitis. This is more common in individuals with heart and lung disease, those with weakened immune systems, infants, and older adults.

Among reports that describe the clinical presentation of patients with confirmed COVID19, most are limited to hospitalized patients with pneumonia. The incubation period is estimated at 4 days (interquartile range: 2 to 7 days). Some studies have estimated a wider range for the incubation period; data for human infection with other coronaviruses (e.g. MERS-COV, SARSCOV) suggest that the incubation period may range from 2-14 days. Frequently reported signs and symptoms of patients admitted to the hospital include fever (77$98 \%)$, cough $(46 \%-82 \%)$, myalgia or fatigue $(11-52 \%)$, and shortness of breath (3-31\%) at illness onset. Among 1,099 hospitalized COVID-19 patients, fever was present in $44 \%$ at hospital admission, and developed in $89 \%$ during hospitalization. Other less commonly reported respiratory symptoms include sore throat, headache, cough with sputum production and/or hemoptysis. Some patients have experienced gastrointestinal symptoms such as diarrhea and nausea prior to developing fever and lower respiratory tract signs and symptoms. The fever course among patients with COVID-19 is not fully understood; it may be prolonged and intermittent. A limited number of reports describe identification of asymptomatic or subclinical infection on the basis of detection of SARS-CoV-2 RNA or live virus from throat swab specimens of contacts of confirmed patients.

Managing an infectious disease and at the same time keeping the economic and social impact comparatively low might include (voluntary) tracing apps to more efficiently control the spread of a virus right from the beginning [5]. Where possible, organisations might increasingly replace face-toface meetings by online meetings and virtual mobility. In many countries, COVID-19 has pushed the use of online tools to manage everyday tasks (e.g. teaching, meeting, planning, home office). These examples have demonstrated how such tools could be used more efficiently not only at times of crisis. Indeed, changing mobility patterns with a greater emphasis of virtual mobility could also contribute to reduce other problems such as climate change by reducing carbon emissions.

However, it is less the technical support but the attitudinal change, which will be required. Even though the pressure is growing to scale down restrictions to public life, it is not yet clear when and whether COVID-19 will fully disappear. The way back to normal life might be longer than originally ex- 
pected and demand significant changes of ethical standards and behavioural patterns regarding both hygiene and individual management of illness more generally. Responsibility and greater awareness of distant but potentially vulnerable others are crucial.

\section{Infection}

When SARS-CoV-2 infects a person, the lesions are not limited to the lungs [6]. The virus causes viraemia after entering the body and the main clinical manifestations are fever, pharyngalgia, fatigue, diarrhoea and other non-specific symptoms. This process includes the incubation phase and the early phase of the disease. The incubation takes 1-14 days (3-7 days being common). Peripheral blood leucocytes and lymphocytes are not significantly reduced (normal or slightly lower) at this phase. Then, the viruses spread through the bloodstream and mainly in the lungs, gastrointestinal tract, and heart, presumably concentrated in the tissues expressing ACE2, the receptor of SARS-CoV-2. This phase occurs around 7-14 after the onset of the symptoms when the virus starts a second attack, which is also the main cause of the aggravation of symptoms. At this time, pulmonary lesions became worse, and chest $\mathrm{CT}$ scans show imaging changes consistent with COVID-19. At this stage, the peripheral blood lymphocytes decrease significantly, involving both $T$ and $B$ lymphocytes. Inflammatory factors in peripheral blood are increased.

Many people may have contracted the virus; however, because they do not develop the symptoms of the disease, they do not know that they are infected and, thus, run the risk of accidently contaminating other people [7]. On the other hand, many are fighting for their lives in hospitals. At the beginning of the pandemic, the fact that the virus is most deadly for the elderly may have imparted the impression that children and young people would not need to be as careful. However, after reports of infection and death of young patients, the mistake was realized. The pandemic is aimed at humanity, but the virus does not affect everyone the same, as it depends on biological, political, economic, cultural, and subjective conditions, actions, and responses.

The unprecedented response by health systems and government at all levels prompted by the emergence and rapid spread of the coronavirus has focused on the heroic efforts of hospital staff to save lives [8]. In contrast, comparatively little attention has been paid to the care needs of the nine out of 10 adults who survive hospitalizations and return to their homes. Most of these survivors are older adults who are coping with multiple chronic conditions and social vulnerabilities and whose health status is substantially complicated by the devastating impact of this virus. For many, early evidence suggests that complex and long-term physical, functional, cognitive, and emotional negative health consequences will be the norm. However, the trajectories of healthcare needs of older adults with COVID-19 in the weeks and months following hospital discharge have yet to be identified. Nor are the challenges that their needs pose for family caregivers well understood. This essential knowledge will develop with time. In the nearer term, the core components of the Transitional Care Model demonstrated to effectively address the holistic needs of "at risk" older adults, coupled with early findings regarding the unique concerns of those with COVID-19, suggest a path for immediate practice and policy responses to caring for this population as they transition back from the hospital to the community.

\section{Pregnant Women and Newborns}

During the COVID-19 pandemic, pregnant women and newborns represent a vulnerable population [9]. To date, although there is no strong evidence-based information showing if pregnant women have a greater chance of getting sick from COVID-19 than the general public, it is well established that pregnant women are at greater risk for severe illness with viruses from the same family as COVID-19 and other viral respiratory infections, such as influenza. Additionally, some experts believe that the reported cases thus far are too limited to be convincing about virulence of COVID-19 infection among this population.

Among this population, cardiopulmonary adaptive alters present during pregnancy (e.g. elevated heart rate and stroke volume, and reduced pulmonary residual capacity) which may increase the risk of hypoxemia and contribute to the increased severity. Thus, health care providers should consider pregnant women and their infants as a high-risk population in control and prevention strategies during pulmonaryassociated pandemics like COVID-19. One of the main concerns associated with COVID-19 is the potential impact on the newborn and its detrimental consequences. Concerns about how to care for a baby after birth, such as breastfeeding, vaccination, or neonatal screening, are among the major concerns of mothers during COVID-19 pandemic.

Maternal-neonatal transmission of respiratory viruses occurs mainly through close contact, transmission through droplets (caregivers, family members, and family visitors), hospital-acquired infections, and exposure to sources of infection from public places. Currently, it is unclear if COVID-19 can cross through the transplacental route to the fetus. In limited case series reported to date, no evidence of virus has been found in the breast milk of women infected with COVID-19 that can be transmitted through breast milk (i.e. presence of SARS-CoV-2 in the breast milk). Similar to their mothers, newborns appear to be a high-risk population against COVID-19 infection due to weakened immune systems. Additionally, potential transmission of SARS-CoV-2 infectious between infected newborns raises another concern during this pandemic.

\section{Nurses}

Healthcare workers, particularly nurses, are at high risk of being infected because they are in the closest proximity to patients [10]. The pandemic of COVID-19 is stressful for healthcare workers. Current research has already shown that healthcare professionals suffer from psychological distress due to COVID-19 pandemic. They experience considerable psychological distress when working with patients diagnosed with COVID-19, including social isolation, role conflicts, fear, and anxiety. 
The fear and anxiety caused by an infectious disease can be devastating if not appropriately identified and managed. Due to the sudden outbreak of the disease, we do not know what nurses experience when caring for patients with COVID-19, especially during the situation where nurses are sent away from their usual work environments to work in the epicenter. These nurses have been working in the epicenters for more than a month now. It is essential to understand the psychological changes in these nurses and ways to manage their stress. This information is urgently needed by nurse leaders to design plans for stress management and interventions to maintain nurses' psychological well-being. The purpose of this study is to examine the psychological experience and change process of nurses in the epicenter of COVID-19 and to provide strategies that nurses could use to handle their stress.

\section{Team Nursing}

The COVID-19 pandemic was declared by the World Health Organization on 11 March 2020 [11]. The rapid spread of SARS-CoV-2 has required an equally rapid response from health-care organizations to find innovative ways to prepare the workforce to care for people with COVID-19.

Working in teams to better utilize the workforce is not a new concept in health care. The Team Nursing model was established in the 1950s and was developed to safely utilize the available nursing skill mix and progress less experienced staff in a safe way. Over time, however, critical care nursing has returned to a focus on individual bedside care.

Collaborative working in a time of a crisis is identified as the key to a successful response. Equally, collaborative practice in health care is recognized as essential in providing excellence in patient-centered care. Despite this, evidence exists of professions working in silos, fragmented as a result of their profession sizes and their position within organizational structures.

The COVID-19 pandemic has resulted in a surge of critical care patients, forcing hospitals to spread their staffing resources and redeploy staff from other areas into critical care. Leaders have been compelled to review their existing workforce and skill mix to rapidly meet the demand of these patients. At the study site the AHP (Allied Health Professions) and nursing teams responded collaboratively, combining the workforce to create a new, interprofessional team of mixed experience, known as the Essential Care Team (ECT). As the pandemic has progressed, much learning has come from the innovation of the ECT and new ways of working have been identified for continued practice and in readiness for a second surge. An evaluative case study of the collaborative AHP and nursing response to COVID-19 is presented.

\section{Role of the Nurse}

The first and foremost role of the nurse is to protect themselves from contracting or transmitting COVID-19 [12]. Adequate personal protective equipment (PPE) should be provided to all health care workers, especially emergency nurses. Even when caring for patients who are critically ill, it is important to protect one's self and take the time to don the appropriate PPE, despite instincts to want to render aid immediately. Education and training around appropriate donning (putting on) and doffing (removing) of PPE is essential, as these are times when health care workers are at a high risk of accidental contamination.

Patients with known or suspected COVID-19 should be placed under appropriate isolation precautions on arrival. Ideally, all patients with COVID-19 would be seen in negative pressure rooms with health care workers wearing airborne precautions (ie, an N95 mask or powered air-purifying respirator [PAPR]). This has proven to be impossible in most areas because of the limited number of these resources. In general, airborne precautions are required for patients with known COVID-19 undergoing aerosolizing procedures, such as cardiopulmonary resuscitation or intubation. Other PPE requirements will be based on local incidence, resources, and local and national guidance. Nurses should review their hospital's policy on what level and type of PPE are required for which risk-level patients. Many hospitals have begun reusing previously single-use PPE, as noted by CDC. CDC has also released a recommendation regarding mask sterilization, which many hospitals are now trialing. Mask sterilization is a process of cleaning and reusing what were previously single-use masks.

Inadequate PPE, the reuse of PPE, and mask sterilization can cause great anxiety for nursing staff as they are concerned for their health and safety. Transparency regarding PPE supply and clear communication regarding reuse and storage of PPE are important for hospital leadership.

In patients with known or suspected COVID-19, both the number of people in the room and the time spent in the room should be minimized. Therefore, several hospitals have placed visitor restrictions. However, despite this, many hospitals have added innovative uses of technology for patients to communicate with family and friends. An example of this would be tablet devices and use of online meeting or communication applications. In addition, many nursing and medical schools have removed students/learners from clinical experiences to reduce exposure and PPE use. Nurses should focus on clustering care as much as possible to limit their time in the room and the number of times they enter and exit rooms. To further cluster care, patients should be given a way to communicate with their care team that does not involve entering the room; this may include a call bell system, a room phone, or a tablet device. It is important to communicate clear expectations for clustered care to patients, as patients may already feel isolated, but nurses also need to protect themselves from unnecessary exposure. Another way to lessen anxiety and loneliness of patients is to have identifiers on PPE. For example, a disposable name tag or permanent names on face shields.

Nurses are part of society, too-the COVID-19 pandemic has rapidly disrupted our everyday lives [13]. We do not need to get sick to be rocked by a radical change in reality. Uncertainty about the future, fear for ourselves and our loved ones, and stressful difficulties, such as unemployment, unstable finances, feelings of isolation, and loss, are all around us. 
As frontline health care professionals, nurses are the true heroes who risk everything to care for patients. They are an important part of health systems, as they handle this crisis and develop strategic planning processes for the future. These processes set objectives, allocate resources, and establish policies that are the framework of the organization and will determine the degree of financial success and quality of care. Nurses will continue to provide care and do the right thing, even in the face of fear and concerns about safety.

During this time of fear and stress, practicing nurses have worked long shifts. Despite the stress and anxiety generated by the workplace environment, they are often not provided with adequate emotional support. The physical and mental exhaustion, lack of knowledge, and in many cases, lack of skills to care for patients can lead to negative emotions and psychological trauma. Under these conditions, the WHO recognizes the importance of nurses' mental health and well-being. Therefore, nurses need supportive psychological interventions to promote emotional release and improve their mental health.

\section{Management}

Because the appearance of the coronavirus is currently the world's biggest health problem, it is necessary to help everyone, and especially the most vulnerable members of society [14]. First of all, additional investments in medical scientific research to get vaccines and medicaments as soon as possible. In this regard, there are several serious announcements that some vaccines and some drugs are in the stages of clinical trials. The appeanance of the coronavirus virus has a very negative impact on the world economy. In order to better protect people from the risk of poverty and social exclusion, who rely on the economic assistance of their countries and to ensure the continuity of support during times of social distancing, countries should adapt their support programs.

The lived experiences, strategies, and policies related to confronting, exploring, and managing COVID-19 are important to healthcare professionals and nurses in preventing and managing future outbreaks of infectious diseases like COVID-19 [15]. In addition to basic and clinical studies, conducting studies on disease-spread prevention and the experience of supporting patients physically and psychologically will be crucial. Examining the effects of administrative strategies on disease-spread prevention in both healthcare and community settings will be valuable. Exploring the impact of cultural differences on the perception and prevention of COVID-19 will be one of the most significant issues related to the spread and management of COVID-19 across countries and cultures.

Furthermore, exploring the psychological distress and social burdens experienced by healthcare professionals and nurses during this outbreak will be vital as well. Better understanding the perceptions, stress, and concerns of nursing and other healthcare professionals may provide critical new information that administrative systems may use to better support these professionals during future infectious disease outbreaks.

\section{Conclusion}

Currently available epidemiological data indicate that the virus of COVID-19 spreads relatively quickly and easily among humans, and it is estimated that one infected person could infect two to three susceptible individuals on average. However, the number of new infections can be significantly affected by a series of preventive measures that we must all responsibly adhere to. Despite the difficult situation, the announcement of the coming vaccines still offers hope. Nurses are most often in contact with COVID-19 patients by making enormous efforts in their treatment. It must never be forgotten.

\section{References}

1. Zhou W (2020) The coronavirus prevention handbook - 101 science-based tips that could save your life. Skyhorse Publishing, New York, USA, 24-27.

2. Baker R (2007) Epidemic - the past, present and future of the diseases that made us. Vision Paperbacks, London, UK, 42-43.

3. Gao GF (2007) Peptide Inhibitors Targeting Virus-cell Fusion In Class I Enveloped Viruses. In: Torrence PF, Combating the Threat of Pandemic Influenza - Drug Discovery Approaches. John Wiley \& Sons, Inc., Hoboken, USA, 226-227.

4. Alipio M (2020) 2019-nCOV Scare: Situation report, role of healthcare professionals and clinical findings. International Journal of Multidisciplinary Health Sciences Research 8: 1-6.

5. Jens O Zinn (2020) A monstrous threat: How a state of exception turns into a 'new normal'. Journal of Risk Research 23: 10831091.

6. Taisheng Li, Hongzhou Lu, Zhang W (2020) Clinical observation and management of COVID-19 patients. Emerg Microbes Infect 9: 687-690.

7. Arruda DP (2020) Coronavirus, inequality and indifference. J Qual Healthcare Eco 3: 000161.

8. Naylor MD, Hirschman KB, McCauley K (2020) Meeting thetransitional care needs of older adults with COVID-19. Journal of $\mathrm{Ag}$ ing \& Social Policy 32: 387-395.

9. Sighaldeh SS, Kalan ME (2020) Care of newborns born to mothers with COVID-19 infection: A review of existing evidence. The Journal of Maternal-Fetal \& Neonatal Medicine 1-13.

10. Zhang Y, Wei L, Li H, et al. (2020) The psychological change process of frontline nurses caring for patients with COVID-19 during its outbreak. Issues in Mental Health Nursing 41: 525-530.

11. Hales P, White A, Eden A, et al. (2020) A case study of a collaborative allied health and nursing crisis response. Journal of Interprofessional Care 34: 614-621.

12. Deitrick K, Adams J, Davis J (2020) Emergency nursing care of patients with novel coronavirus disease 2019. Journal of Emergency Nursing 46: 748-759.

13. Schutz V, Shattell M (2020) Impact of COVID-19: What does it mean for nurses and health systems? Journal of Psychosocial Nursing and Mental Health Services 58: 2-3.

14. Siniša $F(2020)$ The consequences of the corona crisis will be enormous. Med J Clin Trials Case Stud 4: 000255.

15. Chen SC, Lai YH, Tsay SL (2020) Nursing perspectives on the impacts of COVID-19. The Journal of Nursing Research 28: e85. 